\title{
Functional connectivity in the mouse brain imaged by B-mode photoacoustic microscopy
}

Mohammadreza Nasiriavanaki, Wenxin Xing, Jun Xia, Lihong V. Wang

Mohammadreza Nasiriavanaki, Wenxin Xing, Jun Xia, Lihong V. Wang, "Functional connectivity in the mouse brain imaged by B-mode photoacoustic microscopy," Proc. SPIE 8943, Photons Plus Ultrasound: Imaging and Sensing 2014, 894364 (3 March 2014); doi: 10.1117/12.2036638

SPIE. Event: SPIE BiOS, 2014, San Francisco, California, United States 


\title{
Functional connectivity in the mouse brain imaged by B-mode photoacoustic microscopy
}

\author{
Mohammadreza Nasiriavanaki, Wenxin Xing, Jun Xia, and Lihong V. Wang * \\ ${ }^{a}$ Optical Imaging Laboratory, Department of Biomedical Engineering, Washington University in St. Louis, St. Louis, MO 63130, USA \\ * Corresponding author. Email: LHWANG@WUSTL.EDU
}

\begin{abstract}
The increasing use of mouse models for human brain disease studies, coupled with the fact that existing functional imaging modalities cannot be easily applied to mice, presents an emerging need for a new functional imaging modality. Utilizing acoustic-resolution photoacoustic microscopy (AR-PAM), we imaged spontaneous cerebral hemodynamic fluctuations and their associated functional connections in the mouse brain. The images were acquired noninvasively in B-scan mode with a fast frame rate, a large field of view, and a high spatial resolution. At a location relative to the bregma 0 , correlations were investigated inter-hemispherically between bilaterally homologous regions, as well as intra-hemispherically within the same functional regions. The functional connectivity in different functional regions was studied. The locations of these regions agreed well with the Paxinos mouse brain atlas. The functional connectivity map obtained in this study can then be used in the investigation of brain disorders such as stroke, Alzheimer's, schizophrenia, multiple sclerosis, autism, and epilepsy. Our experiments show that photoacoustic microscopy is capable to detect connectivities between different functional regions in B-scan mode, promising a powerful functional imaging modality for future brain research.
\end{abstract}

Keywords: B-scan mode, Resting-state functional connectivity, Functional imaging, Acoustic-resolution photoacoustic microscopy.

\section{INTRODUCTION}

Functional magnetic resonance imaging (fMRI), the most common neuroimaging modality for human brain imaging, requires a very high magnetic field in order to obtain a sufficient signal to noise ratio (SNR) and spatial resolution for small animal imaging ${ }^{1}$. Functional connectivity mapping with optical intrinsic signal imaging (fcOIS) was recently introduced as an alternative method to image functional connectivity in mice ${ }^{2,3}$. In fcOIS, changes in local hemoglobin concentrations are determined based on changes in the reflected light intensity from the surface of the brain ${ }^{2,4}$. Therefore, neuronal activity can be measured through the neurovascular response, similar to the method used in fMRI. However, due to the diffusion of light in tissue, the spatial resolution of fcOIS is limited ${ }^{2,5}$, and the experiment has, thus far, been performed using an exposed skull preparation, which adds complexity for longitudinal imaging. Photoacoustic imaging is an emerging technique that is based on the acoustic detection of optical absorption from tissue chromophores, such as oxy-hemoglobin $\left(\mathrm{HbO}_{2}\right)$ and deoxy-hemoglobin $(\mathrm{Hb})^{6}$. This hybrid nature makes photoacoustic tomography capable of providing high resolution images of the brain while leaving the scalp intact ${ }^{7}$. In Figure 1, graphs of molar extinction coefficients of oxy $\left(\mathrm{HbO}_{2}\right)$ and deoxy $(\mathrm{Hb})$ hemoglobin for wavelengths ranging from $250 \mathrm{~nm}$ to $950 \mathrm{~nm}$ are shown.

Photons Plus Ultrasound: Imaging and Sensing 2014, edited by Alexander A. Oraevsky, Lihong V. Wang, Proc. of SPIE Vol. 8943, 894364 - (C) 2014 SPIE · CCC code: 1605-7422/14/\$18 - doi: 10.1117/12.2036638 


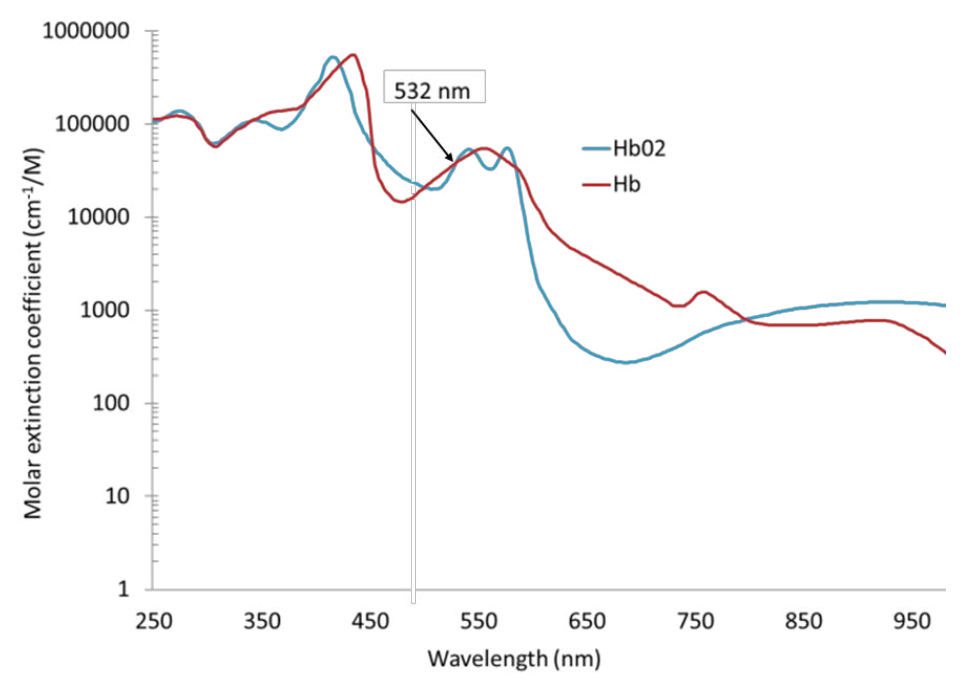

Figure 1. Graph showing molar extinction coefficients of oxy $\left(\mathrm{HbO}_{2}\right)$ and deoxy $(\mathrm{Hb})$ hemoglobin. The molar extinction coefficients of $\mathrm{HbO} 2$ and $\mathrm{Hb}$ are identical at $532 \mathrm{~nm}$.

Previously ${ }^{8}$, we developed a functional connectivity photoacoustic tomography (fcPAT) system, which, for the first time, allowed noninvasive imaging of resting-state functional connectivity in the mouse brain, with a large field of view and a high spatial resolution. Bilateral correlations were observed in eight functional regions, including the olfactory bulb, limbic, parietal, somatosensory, retrosplenial, visual, motor, and temporal regions, as well as in several subregions.

Here, we image the mouse brain in B-mode, and study the functional connectivity between the homologous functional regions in the left and right hemispheres of the mouse brain, non-invasively. We utilize acousticresolution photoacoustic microscopy (AR-PAM) imaging system to image the mouse brain non-invasively. The lateral and axial resolutions are $45 \mu \mathrm{m}$ and $30 \mu \mathrm{m}$, and the focal zone is around $+0.3 \mathrm{~mm} \sim-0.7 \mathrm{~mm}$. By changing the transducer or the numerical aperture, the spatial resolution and the maximum imaging depth of the AR-PAM are saleable within the reach of the excitation photons. An imaging depth of more than $3 \mathrm{~mm}$ in live animals has already been demonstrated using our AR-PAM ${ }^{9}$. This is much larger penetration depth compared to other optical imaging modalities such as optical coherence tomography ${ }^{10,11} 10$. The acquisition time in this system is $250 \mathrm{~ms}$. A typical image acquired by the AR-PAM from the mouse brain is given in Figure 2.

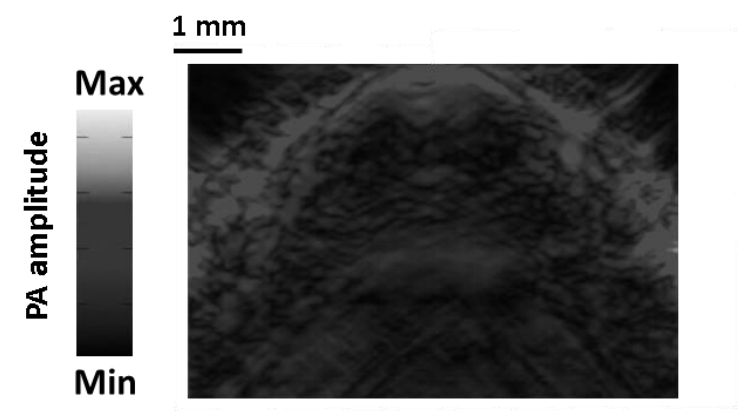

Figure 2. B-scan image of a mouse brain (Bregma 0) acquired non-invasively using the AR-PAM. 
For the experiments, we used 3-4 month old Swiss Webster mice. Before imaging, the animal was briefly anesthetized with $2 \%$ isoflurane, and the hair was removed with a hair remover lotion. The animal was then mounted on the imaging system. We changed the anesthesia from isoflurane to the mixture of ketamine and xylazine, because they provide stronger brain activity. $100 \mathrm{mg} / \mathrm{kg}$ ketamine and $10 \mathrm{mg} / \mathrm{kg}$ xylazine were mixed and administered intraperitoneally. For brain imaging, all experimental animal procedures were carried out in conformity with the guidelines of the US National Institutes of Health. The laboratory animal protocols for this work were in accordance with those approved by the Animal Studies Committee of Washington University in St. Louis.

\section{SEED-BASED ANALYSIS OF FUNCTIONAL CONNECTIVITY}

In each experiment, the animal was imaged using the AR-PAM system for 5 minutes, generating 1200 temporal images. To process the images, the seed-based method is utilized. For the seed-based analysis, the images are processed in order with the following procedures: (a) Smoothing, (b) DC removal, (d) resting-state functional connectivity frequency components (between 0.009 and $0.08 \mathrm{~Hz}$ ) selection, and (e) global regression. The regression algorithm is performed following the procedure described in [23]. Briefly speaking, for a brain data $B$ including $s$ temporal images and $m \times n$ pixels in each image, the global signal is $B_{g}=g^{-1} \times B$, where $g$ is a $1 \times s$ vector that contains the average of each brain image, and $g^{-1}=\left(g \times g^{\prime}\right)^{-1} \times g^{\prime}\left(g^{\prime}\right.$ is the transpose of $g$ ). The global signal is then regressed out from each pixel's temporal signal using $B_{p}=B-g \times B_{g}$. In Figure 3, the time traces of seed locations in the left and right secondary motor cortex are shown. As one can see, there is a strong correlation between the signal from these homologous functional regions.

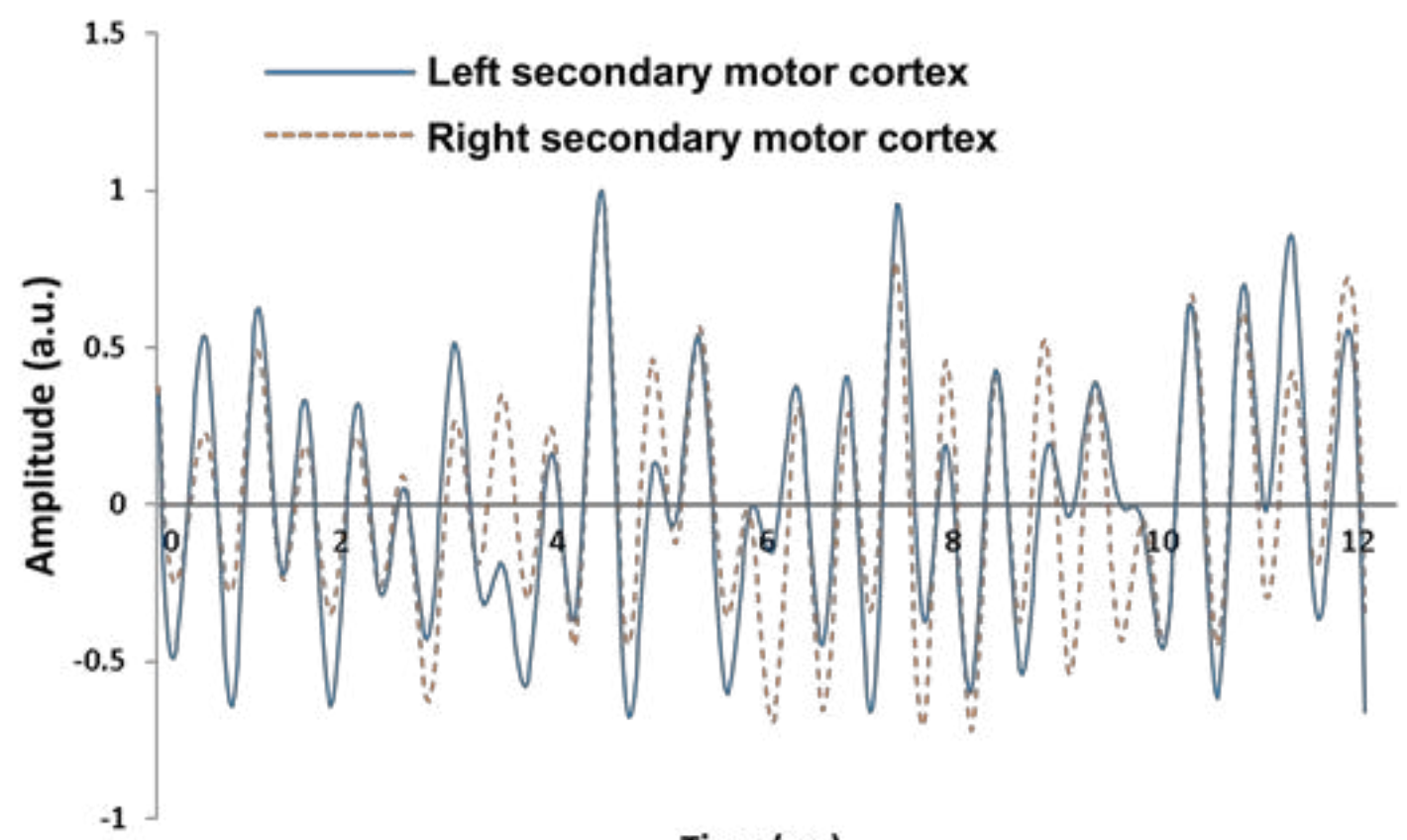

\section{Time (ms)}

Figure 3. Time traces of seed locations in the left and right secondary motor cortex 


\section{RESULTS AND DISCUSSION}

The mice were imaged by using the AR-PAM imaging system. Images were analyzed using the seed-based method as described in section 3. The imaging depth was $3 \mathrm{~mm}$ to $3.5 \mathrm{~mm}$. We co-registered the functional regions on the Paxinos atlas with those on the PAM image. Then, a seed was placed in retrosplenial granular cortex. A strong correlation was observed between the left retrosplenial and right retrosplenial regions (Figure 4). We also observed a strong correlation between the cingulate regions in the left and right hemispheres of the mouse brain. Moreover, simultaneously we observed an anti-correlation between some of the functional regions in left and right hemispheres. We are still investigating the anti-correlated regions. The anti-correlated regions are believed to have opposing functions, but their origins are still being debated. We plan to use electrical stimulation to test the correctness of the results ${ }^{12}$.

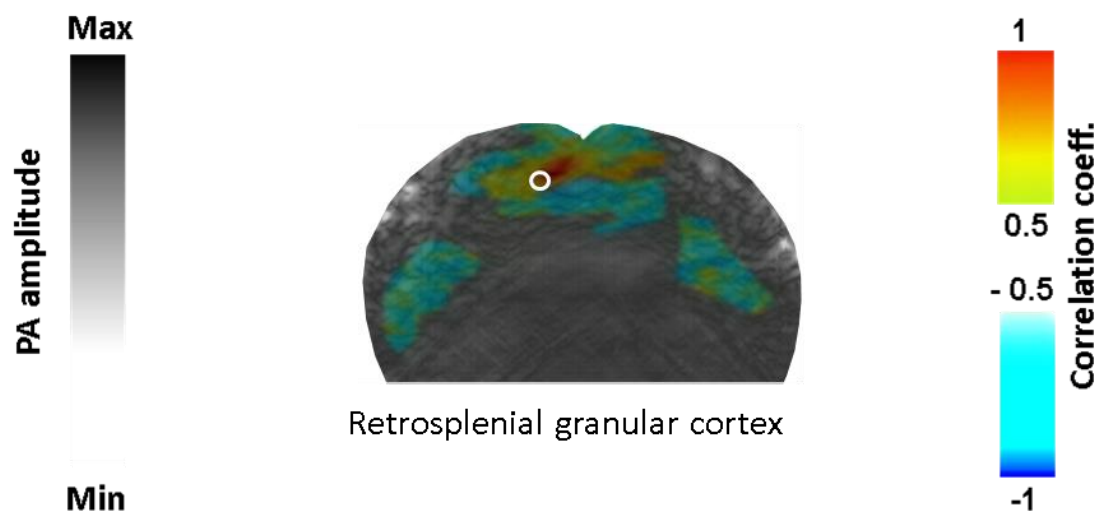

Figure 4. B-mode resting-state functional connectivity maps in a live mouse brain acquired noninvasively by AR-PAM system. Correlation map of retrosplenial granular cortex. White circles: seed regions.

\section{CONCLUSION}

Utilizing acoustic-resolution photoacoustic microscopy (AR-PAM), we imaged spontaneous cerebral hemodynamic fluctuations and their associated functional connections in the mouse brain. The images were acquired noninvasively in B-scan mode with a fast frame rate, a large field of view. At different locations relative to the bregma, correlations can be investigated inter-hemispherically between bilaterally homologous regions, as well as intra-hemispherically within the same functional regions up to several millimeters in depth. The B-scan functional connectivity maps at different brain locations can be combined to eventually form a three dimensional correlation map of the entire mouse brain. These are in our future plan which is ongoing.

\section{ACKNOWLEDGEMENTS}

The authors acknowledge and thank Prof. James Ballard, for his close look at the manuscript. This work was sponsored in part by National Institutes of Health grants R01 EB000712, R01 EB008085, R01 CA134539, R01 CA159959, U54 CA136398, R01 EB010049, and DP1 EB016986 (NIH Director's Pioneer Award). L.W. has a financial interest in Microphotoacoustics, Inc. and Endra, Inc., which, however, did not support this work. 


\section{REFERENCES}

H. Benveniste and S. Blackband, "MR microscopy and high resolution small animal MRI: applications in neuroscience research," Progress in neurobiology 67 (5), 393-420 (2002).

E. Jonckers, J. Van Audekerke, G. De Visscher, A. Van der Linden, and M. Verhoye, "Functional connectivity fMRI of the rodent brain: comparison of functional connectivity networks in rat and mouse," PloS one 6 (4), e18876 (2011).

B.R. White, A.Q. Bauer, A.Z. Snyder, B.L. Schlaggar, J.M. Lee, and J.P. Culver, "Imaging of functional connectivity in the mouse brain," PloS one 6 (1), e16322 (2011).

A.W. Bero, A.Q. Bauer, F.R. Stewart, B.R. White, J.R. Cirrito, M.E. Raichle, J.P. Culver, and D.M. Holtzman, "Bidirectional relationship between functional connectivity and amyloid- $\beta$ deposition in mouse brain," The Journal of Neuroscience 32 (13), 4334-4340 (2012).

S. Bahar, M. Suh, M. Zhao, and T.H. Schwartz, "Intrinsic optical signal imaging of neocortical seizures: the'epileptic dip'," Neuroreport 17 (5), 499-503 (2006).

H. Niu, S. Khadka, F. Tian, Z.J. Lin, C. Lu, C. Zhu, and H. Liu, "Resting-state functional connectivity assessed with two diffuse optical tomographic systems," Journal of biomedical optics 16 (4), 046006-046006-046005 (2011).

L.V. Wang, "Tutorial on photoacoustic microscopy and computed tomography," Selected Topics in Quantum Electronics, IEEE Journal of 14 (1), 171-179 (2008).

J.M. Yang, C. Favazza, R. Chen, J. Yao, X. Cai, K. Maslov, Q. Zhou, K.K. Shung, and L.V. Wang, "Simultaneous functional photoacoustic and ultrasonic endoscopy of internal organs in vivo," Nature Medicine 18 (8), 1297-1302 (2012).

J. Gamelin, A. Maurudis, A. Aguirre, F. Huang, P. Guo, L.V. Wang, and Q. Zhu, "A real-time photoacoustic tomography system for small animals," Optics express 17 (13), 10489-10498 (2009).

Junjie Yao, Jun Xia, Konstantin I. Maslov, Mohammadreza Nasiriavanaki, Vassiliy Tsytsarev, Alexei V. Demchenko, and Lihong V. Wang, "Noninvasive photoacoustic computed tomography of mouse brain metabolism in vivo," Neuroimage $\mathbf{6 4}$ (0), 257-266 (2013).

Mohammadreza Nasiriavanaki, Jun Xia, Hanlin Wan, Adam Quentin Bauer, Joseph P Culver, and Lihong V Wang, "Highresolution photoacoustic tomography of resting-state functional connectivity in the mouse brain," Proceedings of the National Academy of Sciences 111 (1), 21-26 (2014).

Hao F Zhang, Konstantin Maslov, George Stoica, and Lihong V Wang, "Functional photoacoustic microscopy for highresolution and noninvasive in vivo imaging," Nature biotechnology 24 (7), 848-851 (2006).

3 Mohammad Avanaki, Adrian Gh Podoleanu, Mark C Price, Serena A Corr, and SA Hojjatoleslami, "Two applications of solid phantoms in performance assessment of optical coherence tomography systems," Applied optics 52 (29), 7054-7061 (2013).

4 Mohammad Avanaki, Adrian Gh Podoleanu, John B Schofield, Carole Jones, Manu Sira, Yan Liu, and Ali Hojjat, "Quantitative evaluation of scattering in optical coherence tomography skin images using the extended Huygens-Fresnel theorem," Applied optics 52 (8), 1574-1580 (2013).

5 Mohammadreza Nasiriavanaki, Jun Xia, and Lihong V. Wang. "High resolution functional photoacoustic computed tomography of the mouse brain during electrical stimulation." In SPIE BiOS, pp. 85813K-85813K. International Society for Optics and Photonics, (2013). 